\title{
L'usage des marqueurs discursifs ben et be chez des jeunes à l'île Maurice
}

\author{
Shimeen-Khan Chady ${ }^{*}$ \\ CEPED, Université Paris Descartes, 45 rue des Saints-Pères, 75006 Paris, France
}

\begin{abstract}
Résumé. Cet article propose une présentation des usages des marqueurs discursifs « be » et «ben » chez un groupe de jeunes locuteurs mauriciens âgés de 16 à 19 ans. Nous émettons l'hypothèse que «ben », marqueur discursif récemment emprunté au français de France participe de la construction d'un parler hybride caractéristique de certains jeunes. Les analyses reposent sur un corpus de $6 \mathrm{~h}$ de conversations recueillies en 2014. L'analyse fine des interactions montrera d'une part comment ces termes sont associés à différents groupes et différentes situations et d'autre part comment ces associations sont réinvesties en interaction pour des mises en scène du discours.
\end{abstract}

\begin{abstract}
The use of discourse markers "ben" and "be" by young Mauritians. In this paper, we will explore the use of discourse markers "be" and "ben" by a group of young Mauritians aged 16-19. We argue that the discourse marker "ben", recently borrowed from spoken French (from France) contributes to a particular hybrid way of talking among some young people. The analysis is based on a corpus of 6 hours of conversational data collected in 2014. A detailed interactional analysis shows how these markers are associated with different groups and situations and by the same time are contextually reinvested for discourse strategies.
\end{abstract}

\section{Introduction}

Les langues en présence à l'île Maurice offrent différentes possibilités conversationnelles aux jeunes. Nous observons notamment récemment une présence de plus en plus fréquente de formes françaises familières dans leurs usages. Ces nouvelles formes contribuent à renverser la traditionnelle diglossie attribuée aux situations créolophones (Ledegen, 2003 ; Carpooran, 2007). En effet, alors que le français était réservé à un usage formel et les variétés régionales de français empruntaient surtout au créole, nous constatons l'usage de termes familiers empruntés au français de France comme par exemple différentes insultes ou termes argotiques. Nous relevons notamment l'usage de marqueurs discursifs empruntés au français de France comme ben, qui sera l'objet de notre discussion ici. Nous adoptons la définition de Schiffrin des marqueurs discursifs (désormais MD) qui les considère comme des éléments séquentiellement dépendants : «markers propose the contextual coordinates

*chadyshim@gmail.com 
within which an utterance is produced and designed to be interpreted » (Schiffrin, 1987 : 315).

Nous sommes ici dans une perspective de contacts des langues. Notre hypothèse de départ était que le MD ben participe de la construction d'un nouveau "parler », hybride, caractéristique de certains jeunes ${ }^{1}$ à l'île Maurice. Notre analyse montre que ben est utilisé en alternance avec ce qui peut être considéré comme son équivalent créole be et permet aux locuteurs de se mettre en scène à travers leurs discours. Même si ces MD sont, de façon large, associés à différents groupes de locuteurs ainsi qu'à des langues différentes, leurs usages simultanés dans une même conversation montrent des fonctions interactionnelles importantes, ils offrent aux locuteurs différentes possibilités de présenter leurs discours, donnent des informations non négligeables au destinataire quant à l'interprétation du message tout en témoignant de la relation entre les interlocuteurs. Ainsi, l'intérêt de la variabilité de ces MD est discursive et interactionnelle avant d'être identitaire.

Nous commencerons par une brève présentation du cadre de l'étude avant de présenter les données et l'approche. Nous continuerons par quelques recherches portant sur le sujet avant de tenter de montrer comment différentes valeurs, aussi bien discursives que sociales sont associées à ces deux MD et permettent ainsi un usage dynamique en interaction par un groupe particulier de locuteurs jeunes.

\section{Cadre de l'étude}

\subsection{Situation sociolinguistique de l'île Maurice}

La présentation proposée ici servira de repère au lecteur mais nous tenons à préciser que nous avons adopté une approche inductive afin d'éviter une pré-catégorisation des locuteurs et afin de nous focaliser sur ce qui émergeait éventuellement des usages. Nous n'adhérons pas à une vision fixiste de l' " ethnicité » ni de la « langue » et pensons que c'est durant les interactions que les locuteurs construisent et remodèlent les catégories. Nous concédons cependant que les locuteurs se basent sur des ensembles déjà construits et partagés qui leur permettent des réappropriations durant les interactions.

La division de la société mauricienne en ethnies n'est pas aisée puisqu'il n'y a pas de critères réellement pertinents pour les séparer. La classification officielle des groupes ethniques présente trois grands groupes : les Indo-Mauriciens, les Sino-Mauriciens, et la Population Générale. Cependant, ces groupes se basent tantôt sur une origine ancestrale commune (Indo-Mauriciens et Sino-Mauriciens), tantôt sur une religion commune (la Population Générale est chrétienne). Et ces groupes sont eux-mêmes hétérogènes et sousdivisés par des groupes aussi rassemblés selon des caractéristiques diverses comme :

- la religion : parmi les Indo-Mauriciens, on retrouve les Musulmans et les Hindous, euxmêmes divisés en réalité en différentes religions alors que les Sino-Mauriciens regroupent bouddhistes et chrétiens ;

- les traits anthropomorphiques ou le mode de vie : la Population Générale regroupe les Blancs-Mauriciens qui sont descendants de colons et les Créoles, qui sont descendants d'esclaves, sans compter des Indo-Mauriciens chrétiens comme les Tamouls baptisé, les Mulâtres, les Créoles-Chinois (Carpooran, 2013 ; Bosquet-Ballah, 2015).

Il ne s'agit pas de groupes ethniques pouvant clairement être définis et se distinguant des autres par une langue, une religion, une origine ancestrale commune. Cette classification peu convaincante anticipe les difficultés que pourrait poser une approche basée sur un croisement entre formes langagières et groupes ethniques.

Ces groupes utilisent diversement le créole mauricien, le bhojpuri et le français. L'anglais est très faiblement utilisé au quotidien malgré son statut de langue officielle. D'autres 
langues indiennes et chinoises apparaissent surtout comme des symboles identitaires et ne sont vraiment utilisées quotidiennement. On observe à un niveau large et dans les représentations une forme de diglossie : le français et l'anglais sont considérés comme variétés hautes et le créole et le bhojpuri comme variétés basses. Le créole demeure la langue première de la grande majorité. Le français est langue première d'une minorité, les Blancs-Mauriciens mais aussi une partie des Créoles. Le bhojpuri est aussi langue première d'une faible minorité. Au quotidien, ce sont surtout le créole et le français qui partagent les pratiques actives ou passives de la majorité des Mauriciens. Cependant, les interactions montrent généralement que la frontière des langues n'est pas nette, les pratiques interlectales (Prudent, 1981) sont très largement répandues.

\subsection{Pratiques des jeunes à l'île Maurice}

À l'île Maurice, le rapport entre le français et le créole tend à changer chez les jeunes. Carpooran (2007) se demande si nous ne sommes pas face à une "décrispation de la diglossie » comme à la Réunion. Les travaux de Bavoux $(2001 ; 2003)$ et Ledegen (2002 ; 2004) montrent en effet à la Réunion l'apparition d'un registre du français familier chez les jeunes alors que jusqu'à récemment, le français, variété high, ne connaissait qu'un usage courant. Cette nouvelle donne marque une évolution non négligeable dans le rapport diglossique entre les langues. L'usage de MD français dans un discours à dominante française, comme créole, mais aussi de petits mots créoles dans un discours à dominante française, se fait de plus en plus entendre et concourt, d'après nous, à cette décrispation. Signalons en outre l'apparition de formes hybrides créole-français de ces $\mathrm{MD}$, comme à la Réunion (Ledegen, 2013). Nous voyons ainsi émerger une nouvelle dimension oralisée du français chez les locuteurs jeunes, qui contribue, par la même occasion, à de nouvelles dynamiques interactives.

Comme à la Réunion, l'apparition de pratiques sociolangagières propres aux « jeunes » est récente à l'île Maurice et avec celles-ci, de nouveaux usages émergent. Une fois de plus, cette zone, notamment par la nature des contacts linguistiques et tous les enjeux sociaux et représentatifs qui en émanent, permet de saisir au mieux un phénomène (qui ne lui est pourtant pas réservé) par son caractère récent, comme bien souvent les situations créolophones l'ont permis.

Nous nous sommes intéressée, dans le cadre de notre thèse, à la variation de certains de ces MD qui connaissent, a priori, des variantes francisées et créolisées entrant en concurrence dans des fonctions diverses. Nous présenterons ici une partie de cette recherche, en montrant comment ceux-ci contribuent aux jeux conversationnels de jeunes locuteurs mauriciens. Nous nous focaliserons ici sur les résultats concernant be et ben.

\section{Les données et l'approche pluridimensionnelle}

\subsection{Les données}

Ayant adopté une démarche essentiellement inductive, nous sommes partie d'un corpus d'environ 6h recueilli auprès de 9 jeunes âgés de 16 à 19 ans, en 2014. 6 d'entre eux fréquentent la même école, un collège mixte garçons-filles, contrairement à la majorité des collèges de l'île. Ce collège est réputé pour être fréquenté par des francophones (autres que les Blancs-Mauriciens), appartenant à la moyenne bourgeoisie. 5 d'entre ceux de la même école forment une même communauté de pratiques (Eckert, 1989), nous les nommerons le groupe des 5 (G5). Ces 5 jeunes ont pour langue première le français, contrairement à la majorité des Mauriciens. Cependant, arrivés au collège, les garçons utilisent principalement le créole et les filles ont des pratiques plus souples. Les enregistrements obtenus sont ainsi 
aussi bien à langue dominante française que créole.

Soulignons ici que nos données concernent donc un groupe particulier de locuteurs et n'est certainement pas représentatif (et ce n'est pas notre objectif) du reste des jeunes.

Ces jeunes avaient pour objectif d'enregistrer des conversations « ordinaires » entre amis et en famille sans la présence de l'enquêtrice pour pallier au paradoxe de l'observateur (Labov, 1971). Une confrontation aux enregistrements a par la suite été réalisée auprès d'eux. L'enquête repose ainsi sur une analyse de corpus couplée à l'analyse d'entretiens semidirectifs.

\subsection{L'analyse des données}

Nous avons commencé par relever les occurrences des deux « petits mots » dont nous avons analysé les contextes d'apparition en commençant par le contexte langagier, les locuteurs et les contextes des interactions. Cependant, cette première approche ne s'avéra pas pertinente puisque nous observions l'usage des deux formes ben et be dans une même interaction et parfois chez un même locuteur. Nous nous sommes alors plus particulièrement penchée sur les usages conversationnels et avons analysé les occurrences selon le découpage des interactions en séquences ${ }^{2}$. C'est alors que des usages énonciatifs et pragmatiques sont apparus comme essentiels à l'appréhension de la variabilité de ces MD. Les types de discours dans lesquels ils apparaissaient devinrent alors aussi pertinents.

C'est dans cette perspective de croisement entre différents niveaux que nous mobilisons la notion de «mouvements discursifs » de F. François (2005). Nous nous rapprochons aussi des travaux de Vion (1995) et d'Auzanneau et Leclère-Messebel (2007), pour qui les relations entre les locuteurs se construisent dans une interaction selon la conduite que chacun adopte face à l'autre. Nous voyons comment les rapports, qui passent par le langage, émergent et bougent selon différentes configurations qu'il faut considérer en relation les unes avec les autres.

Nous adoptons ainsi une démarche ethnographique et privilégions une analyse fine des interactions ainsi qu'une analyse discursive. Nous nous basons sur des théories sociolinguistiques, interactionnistes mais aussi énonciatives et pragmatiques. Cette approche pluridimensionnelle s'est imposée par notre corpus d'une grande hétérogénéité et nous paraît être la mieux adaptée pour l'appréhension de ce type de phénomènes.

\section{Les marqueurs discursifs chez les jeunes locuteurs}

Même si, comme le précisent Trimaille et Billiez: «les études quantitatives qui permettraient de déterminer des seuils et des effets de seuils manquent » (2007 : 103), il existe quelques études présentant l'usage des MD comme étant une caractéristique du " parler jeune ». En effet, ces usages se retrouvant chez des locuteurs de tout âge, c'est la fréquence d'apparition, ainsi que la co-occurrence des termes qui permettent de les percevoir comme typiques d'un certain âge. Beeching (2010) montre par exemple que le taux des MD, de manière générale, est plus élevé chez les plus jeunes dans un ensemble de trois corpus différents tout en précisant que les usages varient cependant selon les situations. Guérin et Moreno (2015), même si elles n'inscrivent pas ouvertement leur article dans le domaine des parlers jeunes, font néanmoins leur analyse à partir d'un corpus recueilli auprès de jeunes locuteurs. Ce phénomène ne semble, en outre, pas être propre au français puisque Secova remarque aussi la fréquence élevée des mots introducteurs du discours rapporté chez les jeunes aussi bien en anglais qu'en français (corpus recueilli à Londres multiculturel et Paris multiculturel) (2015). Norrby et Winter (2002) présentent le même phénomène en anglais d'Australie et en suédois et Tagliamonte en anglais, chez de jeunes Canadiens (2005). Bien sûr, outre l'âge des locuteurs, certains facteurs semblent influer sur 
la présence de ces MD, Tagliamonte montre par exemple l'influence des relations entre pairs, Guérin et Moreno montrent, quant à elles, l'importance de la connivence entre les interactants.

Une dimension identitaire en milieux plurilingues a en outre été mise en avant par certains chercheurs comme Billiez et Trimaille. Ils expliquent en effet comment les emprunts à l'arabe notamment participent « de la redéfinition des appartenances groupales en milieu urbain » (2007: 5). Chevalier (2014) montre la concurrence entre ben et well dans le chiac du sud-est du Nouveau-Brunswick et Ledegen (2013) présente par exemple le métissage du français et du créole à la Réunion chez les jeunes notamment par l'usage fluide de ces «petits mots » dans des ensembles linguistiques qui vont d'un pôle plus créole à un pôle plus français, avec en outre des termes hybrides comme [Jpakwe] (« je sais pas quoi »), usages que nous pouvons mettre en parallèle avec ceux de Maurice ${ }^{3}$.

Nous voyons ainsi, rien que dans ces quelques études, des approches assez différentes les unes des autres, mais surtout les informations différentes que peuvent véhiculer les «petits mots».

\section{Quelques études sur Ben}

Au niveau interactionnel, Bruxelles et Traverso (2001: 43) montrent que ben n'apparaît jamais en ouverture absolue d'échange et que sa fonction principale est d'annoncer une suite. Ben marque, en situation dialogale, que l'énoncé qu'il accompagne est réactif et en situation monologale, il indique la liaison - l'énoncé est la suite de ce qui précède. Ben permet trois grandes opérations :

- construction du flux (construction thématique et organisation pragmatique du discours) ;

- maintenance du flux (gestion interlocutive, plus précisément par le récepteur) ;

- clôture du flux.

Ben, dans une approche énonciative, en référence à l'article d'A. François (1998) apparaît comme un morphème "paradoxe modal » parce qu'il marque l'engagement du locuteur face à son discours en apparaissant généralement à la charnière d'un diptyque " protase » / « apodose $»^{4}$ (thème / rhème ; question / réponse ; hypothèse / conséquence ; situation / réaction) tout en présentant le discours comme une nécessité objective, et ainsi, en désengageant le locuteur face à l'énoncé. Il marque alors « la fatalité, la conséquence nécessaire, l'embarras et l'atténuation polie, la résignation, l'évidence, etc. » Il explique qu'il y a ainsi un jeu entre engagement et désengagement du locuteur selon la situation pragmatique qui est conditionnée par les enjeux sociaux et les représentations du monde par l'énonciateur.

Dans une approche de contacts de langues, Chevalier (2014) étudie la concurrence entre well et ben en chiac du sud-est du Nouveau-Brunswick chez de jeunes Acadiens. En observant que le chiac n'a retenu que les emplois discursifs de well, Chevalier se demande s'il ne viendrait pas remplir une fonction vacante face à ben. Ses premiers résultats lui permettent d'émettre l'hypothèse d'une spécialisation fonctionnelle malgré les facteurs sociaux qui semblent entrer en jeu.

\section{Ben et $b e^{5}$ dans notre corpus}

Ben comme be apparaissent toujours en rapport à un énoncé autre que celui dans lequel ils apparaissent. Ils ne peuvent être compris indépendamment du contexte d'apparition. Nous nous sommes basée sur les travaux de Bruxelles et Traverso (2001) pour définir les utilisations repérées dans notre corpus. Certaines occurrences apparaissent comme ayant une double fonction. Elles peuvent ainsi marquer : 
- l'accord local qui sert à la co-construction du discours autant sur le plan interactif (je participe à la conversation) que sur le plan discursif (je réponds et je présente un nouvel argument). Dans cette position, ben peut être suivi d'une concession et peut marquer une forme d'évidence ${ }^{6}$ qui remet en question la pertinence de l'énoncé précédent ;

- l'hésitation, à laquelle nous rajoutons qu'il peut parfois marquer une distance par rapport à son propre discours servant ainsi de modalisateur dans une gestion des rapports en interaction afin d'éviter d'atteindre la face de l'interlocuteur. Il est alors souvent accompagné de pauses, d'allongements, ou d'autres procédés de modalisation. Il s'agit souvent d'une réponse à une question qui permet à l'interlocuteur d'entrer dans une structure monologale ;

- dans un conflit en interaction, réaffirmation de son argument premier suite à une faille dans le raisonnement de l'interlocuteur (souvent suivi de « alors »). Il peut alors apparaître comme conclusif de la séquence ;

- changement de plan énonciatif ${ }^{7}$, Bruxelles et Traverso (2001 : 46) notent dans cette catégorie le passage d'un plan énonciatif dans la construction du discours «souvent monologale, mais fortement dialogique, voire polyphonique ». Ben permettrait ainsi de marquer :

- une « envolée émotionnelle»

- un discours rapporté au style direct

- une incise dans le fil du locuteur

Ces fonctions se retrouvent dans notre corpus.

\subsection{Particularités de be}

Be apparaît dans des fonctions que nous ne retrouvons pas avec ben dans notre corpus :

- glissement de thème dans un cadre dialogal mais toujours à partir d'un élément cité plus tôt, que ce soit dans la même conversation ou en référence à une conversation ayant eu lieu précédemment ;

- structuration de son propre discours en situation monologale où il sert de connecteur pour développer ou donner une information supplémentaire sur ce que l'on vient de dire ;

- connecteur concessif où il peut être mis en relation à l'utilisation de mais / me. Cette fonction existe en français de France avec ben mais apparaît dans notre corpus uniquement avec $b e$.

Extrait 1 : Dorian (G5 - 16 ans) et son amie Charlize (16 ans)

[362.11 s] CHA : an::: \ayo j'ai envie d'te faire un câlin là $\backslash$ be selma si mo fer enn twa k- kalin la Dorian mo ka'av gagn enn sak zoure taler la ah:...। (int.) j'ai envie d'te faire un câlin là mais sauf que si je te fais un câlin là Dorian, je peux me faire insulter tout à l'heure

Nous remarquons dans cet extrait la fonction de be comme connecteur concessif, suivi par ailleurs de selma qui renforce ici la concession.

\subsection{Particularités de ben}

Les locuteurs vont jouer des différentes fonctions interactionnelles et valeurs sociolinguistiques pour se mettre en scène et mettre en scène leurs discours. Ces fonctions et valeurs émergent elles-mêmes des usages. Ainsi, ben étant moins utilisé que be, les différents sens sont mis en relief par ces utilisations ponctuelles mais aussi, par opposition à be. Il est nécessaire, afin de comprendre l'utilisation faite par nos locuteurs, dans la 
perspective de mise en scène du discours, de voir rapidement les différentes valeurs sociolinguistiques attribuées aux petits mots en question. C'est ce qui permettra de saisir au mieux toute la portée significative en interaction.

\subsubsection{Ben et les rapports entre interlocuteurs}

Nous remarquons la fréquence élevée de ben chez 2 locuteurs : le père de Coralie dans sa conversation avec sa fille (23 occurrences sur 65 au total) et Farah qui partage la conversation de Tarik (12 occurrences). À eux deux, ils regroupent plus de la moitié des occurrences de ben du corpus. Pourtant Farah parle créole et le père de Coralie français.

Dans l'enregistrement avec Tarik, Farah parle créole mais avec des emprunts au français que nous considérons comme appartenant à une variété prestigieuse du créole. Les « mélanges » de Farah s'éloignent ainsi de ceux des autres locuteurs du corpus. Rappelons par ailleurs qu'il s'agit de sa conversation avec Tarik qui se présente comme étant plus anglophone que francophone, il ne fait pas partie du G5. Tarik présente Farah comme parlant plus créole que français. Ils semblent tous les deux faire partie d'un même groupe à l'école : les musulmans, de façon générale plus créolophones que francophones.

Contrairement au père de Coralie (G5), Farah n'a pas de discours négatif face au créole :

Extrait 2 : Tarik (16 ans) et son amie Farah (16 ans) :

[896.48 s] TAR : ok $\backslash$ e kifer nou'nn- nou aster-la nou pa pe koz angle ou franse $\backslash$ actuellement

okl et pourquoi nou- nous là, nous ne parlons pas anglais ou français| actuellement

[902.30 s] FAR : parski::: m- kreol bokou pli fasil pou koze/ parce que::: m- c'est beancoup plus facile de parler créolel

[906.91 s] TAR : e:: +

$$
\text { et:: }+
$$

[907.91 s] FAR : se inpe kouman nou langage/ natal mem si nou SIpoze koz angle $\wedge$

c'est un peu comme notre langue/ natale même si on est supposés parler anglais $\wedge$

Nous remarquons dans l'extrait précédent les formes « francisées » utilisées par Farah, nous les présentons ci-dessous face aux formes équivalentes « plus créoles » :

Tableau 1 : Formes «francisées » de Farah

\begin{tabular}{|c|c|c|}
\hline Réalisations « francisées » de Farah & Formes « plus créoles » & Traduction française \\
\hline bokou & boukou & beaucoup \\
\hline se inpe & li inpe & c'est un peu \\
\hline kouman & kouma & comme \\
\hline langage & langaz & langue \\
\hline
\end{tabular}

Ainsi, chez elle, l'utilisation de ben apparaît tout de suite comme associée à la "variété francisée » dont elle fait usage. Farah est dans une situation d' « enquêtée » face à Tarik qui mène l'interaction sous forme d'entretien où il pose les questions. Signalons en outre que Farah et Tarik sont dans une salle de classe. On y voit une forme de créole surveillé. 
Contrairement à Farah, la fréquence de ben au niveau interaction / pragmatique chez le père de Coralie (G5) peut s'expliquer par la contrainte situationnelle, nous sommes dans une relation père-fille et il tente tout le long de la conversation de ne pas froisser sa fille lorsqu'il lui donne notamment des conseils pour ses études. Quand elle l'interroge sur leur absence de pratique du créole, en dehors du fait qu'il lui répond que le français et l'anglais sont des « atouts », il lui fait comprendre que ce serait bien qu'elle apprenne une troisième langue. Ben apparaît ainsi en MD qui permet la gestion de la face de l'interlocutrice. Il apparaît ainsi très souvent chez le père accompagné d'autres modalisateurs comme « écoute », « tu sais » qui permettent de nuancer ses propos dans le but de garder une bonne entente entre eux. L'extrait suivant présente ces hésitations et autres modalisations.

Extrait 3 : Coralie (G5 - 17 ans) et son père :

[22.89 s] PER : ben écoute euh: je crois c'est dans la famille $\wedge$

[27.06 s] COR : in

[27.49 s] PER : euh:: moi aussi/ + enfin c'est l'habitude que j'ai eue/ + avec mes parents/ ++ avec ta maman/

[36.83 s] on a tout l'temps parlé le français/ ++

[39.77 s] et:: puis + et:::

[42.59 s] (3.60)

[46.20 s] ben à l'école/

[826.21 s] PER : \{ben écoute tu- écoute tu peux:: tu peux: choisir de pas parler le créole aussi hein/

[896.77 s] PER : \{ben: tu sais c'est parce que c'est une question l'habitude hein// tu vois $\left\{c^{\prime}\right.$ est une question l'habitude/ +

Contrairement à Farah, le père de Coralie (G5) tente de ne pas menacer la face de sa fille, cela correspond au final à une forme d'attention portée à l'élaboration de son discours même s'il ne s'agit pas d'une situation «surveillée » comme on l'entend généralement sociolinguistique.

Nous voyons dans les deux cas que l'enregistreur influe sur la conversation (contrairement à d'autres enregistrements du corpus où les thèmes sont moins sérieux). Nous voyons ainsi apparaître une forme de discours oral français ou francisé, associée à des situations plus ou moins «surveillées» qui passe par l'utilisation de MD provenant du français de France.

Ajoutons que, durant un premier terrain exploratoire, nous avions demandé aux locuteurs de nous raconter des histoires en créole et en français et que be n'apparaissait que 3 fois chez le même locuteur (Damien, qui se présente et est perçu par ses pairs comme plus créolophone) alors que ben apparaissait 7 fois chez 2 locuteurs différents en créole : Coralie (G5), se présentant comme plus francophone et qui pourtant utilise beaucoup plus fréquemment be que ben lors du deuxième terrain, c'est-à-dire lorsque les conditions de production changent et qu'elle se trouve avec des locuteurs dans des situations relâchées et Tarik qui, à l'opposé, se présente comme plus créolophone. Cela appuie notre hypothèse que ben est associé aux situations surveillées.

\subsubsection{Ben : petit mot français}

Outre les usages de certains locuteurs qui présentent ben comme marqueur d'un discours surveillé, que ce soit en créole ou en français, nous voyons des occurrences dans des passages discursifs épilinguistiques (Canut, 2000 ; Culioli, 1997).

Nous avons en effet, comme dans l'extrait suivant, certaines imitations du parler des 
« Blancs » dans lesquelles apparaissent ben :

Extrait 4 : Dorian (G5 - 16 ans), son frère Jordy (18 ans) et ses cousins Christophe (20 ans) et Jean (19 ans)

[148.56 s] JEA : (imitation) qu'est-ce que j'ai remarqué/ ([røma:rke]) les Blancs/ parlent ([pa: $\left.{ }^{: 1]}\right]$ la langue// EUH BEN comme ha alors quand je pars à la chasse ou:: je pars à la pêche/ +++

Les « Blancs » à Maurice constituent le groupe majoritairement francophone. Ici, l'imitation est associée en outre aux pratiques sociales aller à la chasse et à la pêche. En plus de ben, nous avons l'accentuation de la marque d'hésitation française euh. Ben apparaît ainsi comme marqueur d'une variété de français utilisé par ce groupe de locuteurs favorisé et donc à un parler français. Ici, ben n'est pas forcément associé à un discours surveillé mais apparaît en tout cas comme faisant partie de la forme orale de ce groupe. Ils utilisent un français considéré comme la « variété endolingue de prestige », teintée de régionalismes ${ }^{8}$.

Ainsi, un seul croisement des occurrences et des locuteurs ne nous permet pas de saisir la portée de la définition des groupes par l'usage seul de ce petit mot. L'analyse du contexte est nécessaire. Nous voyons ainsi apparaître trois critères différents :

- marqueur du parler des « Blancs » donc d'une variété française qui connaît des caractéristiques particulières

- marqueur d'un discours surveillé hors le groupe « Blancs » par des locuteurs parlant créole dans une forme francisée associé à d'autres formes francisées - marqueur d'un discours surveillé et/ou français en dehors du groupe «Blancs »

Si ben en tant que « petit mot » français ou forme « francisée » semble être attesté, son usage dans des situations surveillées n'est pas systématique. Notons qu'il est en outre absent dans quelques conversations en créole avec très peu de francisations. Par exemple, il n'apparaît pas entre Ethan et sa mère et Ethan insiste sur le fait que sa mère n'a jamais utilisé une autre langue que le créole avec ses enfants. Il n'apparaît pas non plus entre Ethan et son ami qui déclarent n'utiliser que le créole dans leurs interactions.

On ne le relève pas non plus dans la conversation entre Aurélien (G5) et Vincent alors qu'Aurélien utilise pourtant quelques francisations. Nous associons l'absence de ben d'une part à sa proximité avec Vincent et d'autre part au fait qu'ils sont tous les deux des locuteurs masculins. Contrairement à Ethan qui déclare avoir toujours parlé créole chez lui, les parents d'Aurélien (G5) lui parlaient surtout français lorsqu'il était plus jeune. Ils remarquent que celui-ci s'est mis à utiliser plus régulièrement le créole au secondaire. Il s'agit d'une déclaration assez fréquente chez nos locuteurs que nous ne pouvons développer ici mais signalons que la question du genre ainsi que la proximité des locuteurs apparaissent comme des facteurs importants dans l'usage des variétés.

\section{Usages de be}

Rappelons que be est beaucoup plus utilisé que ben dans l'ensemble de notre corpus (491 occurrences de be contre 65 ben). Il apparaît aussi bien dans des conversations à dominante créole que française.

\subsection{Be, petit mot créole}

Face à ben, be apparaît comme un petit mot créole. En effet, nous remarquons que les taux les plus élevés de be apparaissent chez les locuteurs utilisant peu ou pas du tout ben (Ethan, sa mère et son ami, Vincent et Aurélien). Ces mêmes personnes utilisent le créole dans 
leurs enregistrements.

Nous voyons en outre un fort taux d'occurrences chez Stéphanie, qui, même si elle utilise beaucoup le créole dans sa conversation avec Amélie, déclare durant les entretiens utiliser autant le créole que le français. Par ailleurs, Coralie, qui déclare parler uniquement français, l'utilise 24 fois dans sa conversation (à dominante française) avec son copain et 21 fois avec son père. Ces deux locutrices emploient aussi ben.

Les occurrences par locuteurs montrent que be est utilisé par tous à part une locutrice : la mère de Coralie, qui, rappelons-le, ne parle que français et qui, comme son époux, a une représentation dévalorisée du créole mauricien. Dans sa conversation avec Coralie, be n'apparaît pas une seule fois alors que comme nous l'avons mentionné plus haut, Coralie l'utilise pourtant souvent dans d'autres conversations. Nous voyons ainsi comment l'interaction peut elle-même influencer les usages plutôt que les préférences des locuteurs. En effet, nous pensons que l'absence de be de Coralie dans sa discussion avec sa mère vient aussi de leur relation et du fait que sa mère tolère peu les usages du créole. Coralie nous dit en effet dans un précédent enregistrement que si elle parle créole, sa mère la regarde « avec des gros yeux ». Prêtant ainsi attention à ce qu'elle nous dit de sa mère et croisant les occurrences des conversations avec sa mère, son père et son ami Darren, nous arrivons à remarquer comment elle associe be, petit mot créole, à un parler plus relâché. Les différents niveaux d'analyse se superposent bien ici : même s'il s'agit du type de situation (relations entre interlocuteurs, situations plus ou moins relâchées, etc.), un choix est effectué entre une variante plus proche du français et une autre plus créole.

Ainsi, be apparaît beaucoup dans des conversations plus créoles comme celles où Ethan discute avec sa mère (59 occurrences) ou son ami (66 occurrences) mais aussi chez les locuteurs mélangeant plus créole et français.

\subsection{Be dans les situations relâchées}

Outre ce que nous avons présenté ci-dessus avec Coralie, certaines rares mais très signifiantes utilisations de be par Farah et le père de Coralie sont effectuées lors de relâchements de leur part dans les interactions :

Extrait 5 : Coralie (16 ans) et son père

[140.51 s] PER : =c'est p- et c'est pas difficile hein/ enfin c'est: difficile/ pour ceux qui n'apprennent pas

[144.78 s] be je pense si tu t'y mets/ (tape sur quelque chose)

[149.33 s] tu devrais::: ++ s- ++ tu devrais t'y mettrel

[153.76 s] tu devrais t'y- je pense que tu devrais t'y mettre/ parce que:: + je pense ça va compter +

[159.04 s] hein ça \{ce sera $\}$ DÉfinitivement un atout/ pour toi/ +

Il continue ici de conseiller à sa fille Coralie d'apprendre le mandarin et nous voyons apparaître be couplé à un geste (tape sur la table) qui peut marquer un certain relâchement de sa part.

Chez Farah, be apparaît une seule fois vers la fin de l'enregistrement, au bout de 18 minutes. Dans sa conversation avec Tarik, celui-ci a le dessus tout le long: il pose les questions, elle répond et il la contredit parfois ou en lui demande d'approfondir ses réponses. On peut en outre observer qu'il la met mal à l'aise avec certaines questions concernant sa vie amoureuse. Farah paraît hésitante et prend des précautions en utilisant beaucoup de modalisateurs. Dans la réplique suivante, elle est moins hésitante et celle-ci pourrait témoigner de sa fatigue de la conversation. Tarik réagit d'ailleurs très différemment à cette 
intervention de Farah et tente alors de « rattraper» la situation en gérant la face de son interlocutrice.

Extrait 6 : Tarik (16 ans) et son amie Farah (16 ans)

[1068.11 s] TAR : kifer to gagn boukou kas ar sa 1 e to pa bizin save pou to- kan to vinn vie ou kwa pou bann retirement plan\ pourquoi tu gagnes beaucoup d'argent avec çal et tu n'as pas besoin d'épargner pour ton- quand tu seras vieille pour la retraitel

[1073.40 s] FAR : ben oui mais: la vie n'est pas toujours facile/ \{alors zis[1076.48 s] TAR :

\{me dezan

trwazan apre to gagn sis set milion\

deux ans trois ans tu auras six sept millions

\{mais au bout de

[1078.88 s] FAR : we be to lavi prive to nepli ena enn lavi prive ariv enn moman/ oui mais ta vie privée tu n'as plus de vie privée à un moment/

[1082.56 s] TAR : mhm::: to bien mature mhm:: t'es bien mature

Notons d'ailleurs ici que Farah associe ici ben à un énoncé français et be à un énoncé créole.

Nous voyons ainsi comment des différentes situations émergent des sens associés aux différents mots qui sont réutilisés en interaction et permettent de jouer sur la présentation de son discours.

\section{Usages chez notre groupe}

Nous avons montré jusqu'à présent comment les différentes fréquences d'occurrences ainsi que certains usages ponctuels permettaient de comprendre les associations des différents MD à des groupes divers de locuteurs mais aussi, dans d'autres cas, à la situation de communication. Ainsi, les différents niveaux d'analyse mais aussi la mise en relation de l'usage de ben et be avec d'autres «marqueurs » des parlers des locuteurs nous ont permis de cerner au mieux les différentes utilisations et perceptions.

Nous aurons chez le $\mathrm{G} 5^{9}$ :

- des alternances plus fréquentes entre les différents petits mots qui marquent les mouvements discursifs et le caractère fluide des interactions et des êtres parlants ;

- l'utilisation de be dans des discours à dominante française qui apparaît clairement comme un procédé stylistique propre à ce groupe et non uniquement dans des moments de relâchement comme nous avons pu le voir plus haut avec Farah et le père de Coralie ; - ben n'apparaît pas forcément comme associé à une variété prestigieuse.

Les locuteurs se basent plus ou moins consciemment sur les différentes associations vues jusqu'à présent afin d'en faire un usage stylistique. En jouant, au gré de leurs envies et de l'évolution de la communication, sur les différents critères des MD, leurs interactions deviennent plus dynamiques, plus vivantes. C'est ce qui différencie le G5 des autres locuteurs, notamment de Farah et du père de Coralie, chez qui, comme nous l'avons vu, les usages sont quasi uniformes. Les «petits mots » chez le G5 sont réinvestis des différentes dimensions dans le fil de l'interaction. Nous pensons qu'il s'agit là d'une caractéristique de ce groupe de jeunes. Signalons que si cela est possible, c'est parce que les locuteurs partagent les mêmes connaissances des différentes valeurs attribuées aux différentes réalisations et qu'ils forment une même communauté de pratiques (Eckert, 1989). L'approfondissement que propose Nicolaï de la notion du « répertoire » de Gumperz (1971) fait sens pour notre travail. Nicolaï propose un «répertoire non-fini », c'est-à-dire qui 
accepte une certaine souplesse et laisse place à la créativité (Nicolaï, 2012). Leur « parler » stylistique sera marqué par cet usage particulier des petits mots et du caractère hybride de celui-ci.

Signalons que chez le G5, l'alternance des deux formes et le mélange entre français et créole en général ne sont pas dévalorisés, certains qualifient même leur façon de parler français de français «potis », terme qui désigne un hybride français / créole. En qualifiant leur français de la sorte, ils s'approprient un qualificatif péjoratif pour certains pour en faire quelque chose de positif, qui les représente et admettent ainsi complètement leur parler hybride. Certains disent aussi que ces alternances et hybridités sont caractéristiques « des gens comme nous, de la moyenne société » et particulièrement jeunes. On voit bien ici cette décrispation de la diglossie. Cependant, pour d'autres du même groupe, ces mélanges sont surtout acceptés au sein de leur groupe de pairs mais peuvent facilement être associés à un « mauvais » français chez d'autres locuteurs.

Alors qu'une analyse par locuteurs nous a permis dans une certaine mesure de mettre quelques spécificités en avant, nous arrivons ici à la limite de cette approche. Nous remarquons en effet chez les membres du G5, dans leurs conversations avec d'autres membres du groupe, une fréquence relativement similaire des petits mots avec une faible apparition de ben dont les occurrences ne seront pas moins significatives. Le facteur le plus important ici sera celui de l'interaction ${ }^{10}$. Nous avons choisi pour cette partie de présenter quelques extraits afin de montrer les usages chez le G5.

\subsection{Be et ben associés au " parler hybride des locuteurs ॥ et discours rapporté}

Tout d'abord, contrairement à ce que nous avons pu voir jusqu'à présent, ben n'est pas toujours associé à une variété francisée surveillée et les deux petits mots peuvent être utilisés indifféremment dans des mêmes fonctions, par un même locuteur.

Dans l'extrait suivant, Amélie (G5) raconte à Stéphanie (G5) sa dispute avec Anthony, son petit ami. Elle explique qu'elle a vu Anthony en ligne sur Facebook et pense qu'il discutait avec d'autres personnes par messagerie. Anthony, qui vient d'avoir internet sur son portable, lui dit qu'il cherchait un jeu et qu'il ne sait pas comment faire et demande à Amélie de l'aider :

Extrait 7 : Stéphanie (G5 - 16 ans) et Amélie (G5 - 17 ans) :

[57.16 s] AME : là i' me fait (DR) ouais ben je sais pas quoi f- comment faire ça

$$
\text { dis-moi/ explique-moi/ } 」 \begin{aligned}
& \text { sipa kwa } \\
& \text { et tout }{ }^{11}
\end{aligned}
$$

[62.22 s] là je dis (AutoDR) nan be moi aussi je sais pas bon tchat/」 (rires)

[66.91 s] tu- tu me connais/

[68.34 s] (DR) bon tchat \{après on se parle sipa kwa ${ }^{12}$

[69.16 s] STE : $\quad$ to bann foutan la

\{quand tu te fous des gens ${ }^{13}$

[70.62 s] AME : ouais/ +après il me fait (DR) ki bon tchat toi tu es dessus presque

de quoi bon tchat

tous les jours moi je rent' qu'un jour tu me fais la gueule $\rfloor$ sipa

kwa j'ai dit $\{(D R)$ ouais be $\rfloor$

et tout

Nous remarquons en effet dans cet extrait qu'Amélie utilise indifféremment be et ben en ouvreurs du discours rapporté associés à « ouais » ou « nan » ${ }^{14}$.

Signalons la fréquence des petits mots dans ce court extrait, six pour seulement trois 
interventions en une durée de 18 secondes. Alors que ben et be apparaissent dans les changements de plan énonciatif en ouverture de discours direct, nous voyons l'usage de sipa $k w a$, forme hybride, en clôture, à trois reprises. Sipa kwa est une forme hybride dont le $k i$ de sipa ki est substitué par kwa, forme plus proche du français quoi. Le sens est « je ne sais quoi ». Nous retrouvons chez notre groupe l'usage des deux formes ainsi que sipa kete et sipa quelle affaire, autre forme hybride.

Nous remarquons en outre les alternances français / créole par l'intervention de Stéphanie («To bann foutan la » [69.16 s]) et le discours rapporté d'Anthony dans celle d'Amélie en [78.85 s] («ki bon tchat»). Nous voyons bien ainsi comme ces usages font partie de leur façon de parler.

\subsection{Incises sous forme de stylisations parodiques}

Les deux extraits suivants présentent un changement de plan énonciatif de la part de Stéphanie qui utilisera une fois ben et l'autre fois be. Ces deux extraits se suivent de quelques secondes et le thème est le même : Stéphanie émet une supposition sur le cadeau que va lui offrir son petit ami pour la Saint-Valentin. Notons que ces changements de plans énonciatifs sont liés à des émotions de la locutrice qui change par la même occasion son intonation. Nous sommes ici dans des usages que nous pourrions rapprocher de stylisations parodiques (Barbéris, 2005 ; Trimaille, 2007) d'un groupe sociotypique ${ }^{15}$.

Extrait 8 : Stéphanie (16 ans) et son amie Amélie (17 ans)

[102.15 s] STE : moi je crois qu'je vais avoir un- un truc de tie and dye multicolor pour la Saint Valentin/

[106.82 s] AME : vre a'ec Noah/ \{Vincent/ \{(rires) c'est vrai ?

[107.78 s] STE : $\quad$ ouais

[109.47 s] (plus fort) \{Vincent ben (bafouille) Vincent/ oh là là Vincent hm

[112.75 s] AME : a'ec qui tu auras:: tie and dye \Vincent $\backslash$ Noah $\backslash+$

[115.88 s] STE : nan Noah parce que- je sais pas je crois::

Extrait 9 : Stéphanie (16 ans) et son amie Amélie (17 ans)

[146.36 s] STE : be ti a bon c'est ça/ +

ben ce serait bien que ce soit çal +

[147.85 s] i' m'a dit un de tes cadeaux \{ça veut dire j'avais deux cadeaux

[149.35 s] AME : \{tu es pas sûre en fait

[150.72 s] STE : (plus bas et en riant légèrement) je suis pas sûre non

[152.50 s] (plus fort) be ki ena inn zis devine/ + esaye ++

ben qu'est-ce qu'y a, on a juste devinél + essayé ++

Nous voyons ainsi dans l'extrait 1 , ben apparaît aux côtés de oh là là, associé à un parler français, son intervention est en outre marquée par un ton ironique et un haussement de la voix. Cet énoncé a une forte valeur émotive et Stéphanie ne répond pas ici par une déclarative comme on pourrait s'y attendre mais par cette exclamation provoquée par le rappel d'une situation antérieure. Nous avons ici une sorte d'incise.

Dans l'extrait 2, be apparaît en revanche dans une phrase créole qui nous semble aussi être un mimétisme sociotypique associé à un groupe de locuteurs plus créolophones. Stéphanie hausse aussi le ton pour cette intervention et signalons l'usage de inn, qui marque un accompli, sans la présence d'un pronom personnel au lieu de $m o^{\prime} n n$ " j'ai ». L'accompli sans le pronom personnel apparaît comme une forme typique tendant vers le pôle plus créole. Nous sommes aussi dans un énoncé à forte valeur émotive où Stéphanie reproche 
sur le ton de la plaisanterie à son interlocutrice ce qu'elle considère comme une critique.

\subsection{Réactif évidence / opposition}

Les deux extraits suivants sont tirés de la conversation entre Coralie (G5) et son petit ami Darren. Rappelons que Coralie se présente comme francophone et dit ne pas parler créole. Bien qu'elle accuse un nombre élevé de be dans cette conversation (24 sur 38 en tout), nombre qui, une fois de plus, dans ce contexte linguistique français particulier et associé à d'autres éléments, met en avant ce MD créole comme caractéristique du « parler » de notre groupe, nous avons choisi de présenter quelques-uns de ses usages de ben afin de voir les procédés en interaction.

Dans l'extrait 1 , Coralie se trompe de mot pour désigner un terrain à l'abandon, elle utilise le mot «kann » et Darren, qui ne voit pas d'abord de quoi elle parle finit par se moquer d'elle lorsqu'elle ne retrouve plus le mot créole adéquat. Elle se justifie alors en disant qu'elle ne parle pas créole et Darren se moque à nouveau d'elle et c'est là qu'elle utilise ben en réaction à l'intervention de Darren, dans un mouvement d'écart face à la situation, face à Darren, écart marqué ici par le langage. Soulignons que Coralie utilise plus souvent be que ben pas uniquement dans cette conversation mais dans tous les enregistrements que nous avons d'elle : 45 be contre 7 ben.

Extrait 10 : Coralie (G5 - 17 ans) et Darren (19 ans)

[457.38 s] DAR : alors quand c'est des mauvaises herbes c'est d'la canne/

[459.12 s] COR : nan:: mais en créole/ comment on dit ça\dan kann/

[462.63 s] DAR : fatak on dit ça en créole/

[464.34 s] COR : eh sori je parle pas créole

$$
\text { désolée }
$$

[466.13 s] DAR : (se moquant) TOUTswit la (rires)

$$
\text { tout de suite }
$$

[467.89 s] COR : an tu m'as d'jà vue TEnir une conversation en créole/

[470.00 s] DAR : non $\backslash$

[470.51 s] COR : ben alors/ j'arrive pas aussi/

[472.71 s] DAR : ouais tu parles un créole ça mmh::: \{un niveau extrêmel

[475.54 s] COR :

$\{$ (rires) ouais mon créole est

bon $\backslash$ (en riant légèrement) pour mon niveau $\+$

Nous voyons en effet comment Coralie répond à l'intervention de Darren qui insinue le contraire de ce qu'elle dit, soit qu'elle parle créole. Après la réponse négative de Darren en [470.00 s], le ben, associé au français, apparaît dans ce consensus local, en opposition avec ce prétendait Darren plus haut, comme un renforcement du discours. Elle dit qu'elle ne parle pas créole et utilise ici la forme française du petit mot alors qu'elle utilise plus souvent la forme be. Signalons en passant que ce passage peut aussi être interprété en rapport à la présence de l'enregistreur. Nous pouvons en effet nous demander si l'intervention de Coralie en [464.34 s] n'est pas adressée en premier lieu à un allocutaire absent, nous, à qui elle rappelle ses discours précédents dans lesquels elle se présente comme non-créolophone. Cela expliquerait la rapidité avec laquelle Darren lui concède ce point.

Dans ce deuxième extrait, Coralie demande à Darren ce qu'il compte offrir à sa copine (c'est-à-dire elle) pour la Saint-Valentin. Ils évoquent la petite amie de Darren comme une personne tierce dans un jeu conversationnel. Coralie n'est pas satisfaite de la réponse de Darren et les quelques interventions font apparaître un écho des petits mots avec be chez Darren et ben chez Coralie dans un mouvement d'opposition : 
Extrait 11 : Coralie (G5 - 17 ans) et Darren (19 ans)

[1306.58 s] COR : EH la saint valentin vendredi là/ qu'est-ce tu as acheté pour elle/

[1310.39 s] DAR: (en riant) enn pen drive une clé usb

[1312.85 s] COR : ça y est tu as fini d'acheter//

[1314.46 s] DAR : non $\backslash+$

[1315.35 s] COR : combien de gigas tu vas prendre/ +

[1317.28 s] DAR : deux/ +

[1318.35 s] COR : c'est TOUT//

[1319.09 s] DAR : be oui $\backslash$ suffisant/

[1321.15 s] COR : mais elle m'a dit que:: sa carte mémoire est quatre gigas/ +

[1325.45 s] DAR : \{alors $\}$

[1325.52 s] COR : \{donc tu dois prendre plus que quatre gigas/

[1328.04 s] DAR : sa carte mémoire est quatre gigas $\backslash$ je dois prendre plus que quatre gigas $/=$

[1330.35 s] COR : =ben oui/ pour:- pour stocker tous ses file/

[1333.42 s] DAR : be c'est quatre gigas j'achète quatre gigas

[1335.18 s] COR : ben non// si elle a encore à stocker/

[1337.36 s] DAR : be elle mettra sur son laptop qu'elle veut pas faire réparer/

[1340.07 s] COR : (rires)

ordinateur portable

$[1341.70 \mathrm{~s}]$ DAR : fuck ++

merde ++

[1343.46 s] COR : ben non achète seize gigas/

Nous voyons une fois de plus ici comment Coralie s'éloigne de Darren en utilisant ben en écho au be intensifiant le désaccord local.

\section{Conclusion}

Dans les conversations présentées, nous avons montré comment les marqueurs discursifs be et ben se chargeaient de différentes valeurs selon les contextes d'utilisation et comment ces différentes valeurs sont réinvesties en interaction par notre groupe de jeunes locuteurs dans une fonction stylistique. Ces différentes valeurs, pour être interprétables, doivent être partagées par les interlocuteurs, cet élément est primordial pour que la dynamique soit effective. Cette dynamique fonctionne ainsi à des niveaux différents et se traduit, à l'échelle de l'interaction, par des mouvements qui marquent les changements de thème, la relation entre les interlocuteurs, la présentation de soi, les hésitations, les réponses, la distance par rapport à son discours. Cela rappelle ainsi que l'interaction, mettant en scène des locuteurs sensibles, ne peut être linéaire et que les unités de la langue ne prennent sens que par l'énonciation, dans un ensemble contextuel suffisamment riche permettant de les interpréter.

Nous avons pu voir que la faible présence de ben n'en était pas moins significative puisque, témoignant d'une forme de français oralisé récente, elle marque de nouveaux usages et fait sens dans un ensemble de caractéristiques qui permet les auto- et hétérocatégorisation au niveau social et individuel et permettent des jeux conversationnels. Ben connaît des valeurs différentes selon les locuteurs et les situations :

- marqueur d'une situation plus ou moins surveillée que ce soit en français ou en créole ;

- associé à un groupe de locuteurs, à savoir les Blancs.

Il est utilisé dans les interactions de notre groupe des 5 , les valeurs précédentes sousjacentes, dans des objectifs pragmatiques (donne une information sur la façon dont doit être interprété l'énoncé) ou énonciatifs (présente l'attitude du locuteur face à son discours ou à 
celui qu'il rapporte plus ou moins explicitement).

$B e$ apparaît, quant à lui, comme un mot typiquement créole adopté par nos locuteurs jeunes dans leur «parler» qu'il tende du plus créole au plus français en passant par une forme hybride.

Parmi les facteurs qui semblent influer sur l'apparition de ben, nous relevons :

- la proximité entre les locuteurs

- le degré de surveillance de la situation

- le poids de l'ancienne représentation diglossique

- le genre reste une question à creuser.

\section{Conventions de transcription ${ }^{16}$}

Dans la transcription apparaissent les chevauchements de parole, les pauses et les intonations montantes et descendantes. Nous avons été, évidemment, confrontée au choix de la graphie de certains segments influant, malgré nous, sur la catégorisation des langues en présence, problème courant dans ce type de situation de contact, particulièrement pour la transcription de parlers «hybrides » qui mélangent - variétés de - langues ou autres pratiques interlectales (Prudent, 1981). Nous nous sommes basée sur notre sentiment personnel ainsi que sur les discours épilinguistiques recueillis auprès des locuteurs. Notre transcription laisse ainsi apparaître une part interprétative (Mondada, 2000). Pour la transcription de la variation, nous avons choisi d'adapter l'orthographe pour être au plus près de la façon dont les locuteurs catégorisent eux-mêmes la situation, malgré les risques de stigmatisation connus.

Ci-dessous la convention de transcription adoptée :

allongements plus ou moins longs - le nombre de «:» dépend de la longueur de l'allongement (à l'oreille)

$+\quad$ pauses plus ou moins longues - + de 0,5 secondes à 1 seconde -++ de $1 \mathrm{sec}$ à $1,5 \mathrm{sec}$ +++ de 1,5 à $2 \mathrm{sec}-$ au-delà de $2 \mathrm{sec}$, le temps est indiqué en secondes entre parenthèses (mesurées manuellement)

(X) passages incompréhensibles - nombre de «X» selon longueur du passage incompris

(?) doute du transcripteur

( ) commentaires du transcripteur

/ intonation montante

1 intonation descendante

MAJ augmentation de l'intensité

ITA traduction

[ ] indications phonétiques

\{ $\}$ chevauchements arrêt brusque d'un mot

$」 \quad$ fin du discours rapporté

\section{Références bibliographiques}

Auzanneau, M., \& Leclère-Messebel, M. (2007). Variabilité linguistique et positionnements dans des interactions de formation, 219-239.

Baggioni, D., \& Robillard, D. de. (1990). Ile Maurice: une francophonie paradoxale. L'Harmattan.

Barbéris, J.-M. (2005). Le processus dialogique dans les phénomènes de reprise en écho. In Dialogisme et polyphonie. De Boeck Supérieur. 
Bavoux, C. (2001). Pratiques langagières de lycéens réunionnais, pratiques diglossiques? Travaux et Documents, (15), 31-46.

Bavoux, C. (2003). Fin de la « vieille diglossie» réunionnaise ?, Anciens et nouveaux plurilinguismes(2).

Beeching, K. (2010). La co-variation des marqueurs discursifs bon, c'est-à-dire, enfin, hein, quand même, quoi et si vous voulez: une question d'identité ? Langue française, (154), 78-93.

Bosquet-Ballah, M. H. Y. (2015). Langue(s) et espace(s) en contexte plurilingue : le cas de l'île Maurice. Université de la Réunion / Université de Maurice, Île Maurice.

Bruxelles, S., \& Traverso, V. (2001). Ben : apport de la description d'un « petit mot du discours » à l'étude des polylogues. Marges linguistiques, (2), 38-55.

Canut, C. (2000). Subjectivité, imaginaires et fantasmes des langues : la mise en discours "épilinguistique". Langage et société, (93), 71-97.

Carpooran, A. (2007). Les parlers jeunes à Maurice: survol descriptif et esquisse typologique. In Pratiques linguistiques des jeunes en terrains plurilingues. Editions L'Harmattan.

Carpooran, A. (2013). Quelques variétés sociolectales du français de Maurice. In La variation $d u$ français dans les espaces créolophones et francophones: Amérique. L'Harmattan.

Chevalier, G. (2014). La concurrence entre 'ben' et 'well' en chiac du sud-est du Nouveau-Brunswick (Canada). Cahiers de sociolinguistique, (7), 65-81.

Culioli, A. (1997). Subjectivité, invariance et déploiement des formes dans la construction des représentations linguistiques. In Diversité des langues et représentations cognitives (C. Fuchs et S. Robert, p. 43-47).

Eckert, P. (1989). Jocks and Burnouts: Social Categories and Identity in the High School. Teachers College Press.

François, A. (1998). Ben en français oral, l'énonciateur (dés)engagé. Proceedings of the 16th International Congress of Linguists. Pergamon, Oxford, Paper No.0331.

François, F. (2005). Interprétation et dialogue chez des enfants et quelques autres : Recueil d'articles 1988-1995. Lyon: ENS-LSH Editions.

Guérin, E., \& Moreno, A. (2015). Présence/absence de particules d'amorce et de particules d'extension dans le discours rapporté : peut-on parler de variation? Langage et société, (154), 67-82.

Gumperz, J. J. (1971). Language in Social Groups. Stanford University Press.

Labov, W. (1971). The study of language in its social context. In Advances in the Sociology of Language (Fishman J.A., p. 180-202). La Haye, Mouton.

Ledegen, G. (2002). Les « parlers jeunes » à la Réunion : « i totoch sérieux ». Pratiques langagières urbaines - Enjeux identitaires, enjeux cognitifs, (130).

Ledegen, G. (2003). Regards sur l'évolution des mélanges codiques à la Réunion : l'avènement de l'interlecte ? Glottopol, 2 (Anciens et nouveaux plurilinguismes), 101-107.

Ledegen, G. (2004). « Le parlage des jeunes » à la Réunion bilan et perspectives, (9), 9-40.

Ledegen, G. (2013). Contact de langues à La Réunion : "On ne débouche pas des cadeaux. Ben i fé qoué alors ?". Langues et Cité, (16), 9-10.

Ledegen, G., \& Lyche, C. (2012). A la recherche du « pe(r)ler » de 'Meurice' : une étude sociophonologique en zone créolophone. SHS Web of Conferences, 1, 2191-2201.

Mondada, L. (2000). Les effets théoriques des pratiques de transcription. Linx, (42), 131-146.

Nicolaï, R. (2012). Du contact entre les langues au clivage dans la langue. Vers une anthropologie renouvelée. Journal of Language Contact, (5), 279-317.

Norrby, C., \& Winter, J. (2002). Affiliation in Adolescents' Use of Discourse Extenders. In Proceedings of the 2001 Conference of the Australian Linguistic Society. Australia: Cynthia Allen.

Prudent, L.-F. (1981). Diglossie et interlecte. Langages, 15(61), 13-38.

Robillard, D. de. (1992). L'aménagement linguistique du français à l'Île Maurice : un exemple de réconciliation de la théorie et de la pratique ? Présence Francophone, (40), 121-134.

Schiffrin, D. (1987). Discourse Markers. Cambridge University Press.

Secova, M. (2015). Discours direct chez les jeunes : nouvelles structures, nouvelles fonctions. Langage et société, (151), 131-151.

Tagliamonte, S. (2005). So who? Like how? Just what? Journal of Pragmatics, 37(11), 1896-1915.

Trimaille, C. (2007). Stylisation vocale et autres procédés dialogiques dans la socialisation langagière adolescente. Cahiers de praxématique, (49), 183-206.

Trimaille, C., \& Billiez, J. (2007). Pratiques langagières de jeunes urbains : peut-on parler de 
« parler»? In Les français en émergence (Bern, Peter Lang, p. 95-109). C. Molinari et E. Galazzi.

Vion, R. (1995). La gestion pluridimensionnelle du dialogue. Cahiers de linguistique française, (17), 179-203.

\footnotetext{
${ }^{1}$ Une catégorie « jeune » doit bien sûr être questionnée en tant que construction sociale mais ce ne sera pas l'objet de notre discussion ici. Nous définissons notre groupe ici par l'âge et par le fait qu'ils sont au même niveau de classe à l'école.

${ }^{2}$ Nous reprenons le découpage de l'interaction selon Traverso (1999): l'intervention est l'unité monologale, l'échange correspond à la plus petite unité dialogale, la séquence a été définie ici par la thématique et l'interaction constitue l'enregistrement.
}

${ }^{3}$ Signalons qu'à Maurice aussi nous voyons apparaître ce type de forme hybride, sipa ki connaît par exemple aussi une forme hybride sipa quelle affaire (« je ne sais pas quoi » pour une traduction simplifiée).

${ }^{4}$ Il rejoint en cela l'analyse de Bruxelles et Traverso.

${ }^{5}$ Signalons qu'il existe une autre réalisation : abe, qui n'apparaît que deux fois dans notre corpus, chez la mère d'Ethan, qui déclare ne parler que créole. Notons par ailleurs que c'est sous cette forme qu'il apparaît dans le Diksioner Morisien (Carpooran, 2009). Cette forme apparaît pour nous comme " plus créole ».

${ }^{6}$ Pour plus d'information sur ben et l'évidence, se référer à François (1998).

${ }^{7}$ Bruxelles et Traverso (2001:46).

${ }^{8}$ G. Ledegen et C. Lyche (2012 : 2193) rappellent que Baggioni et Robillard (1990) (1992) repèrent à Maurice trois variétés de français :

- la «variété endolingue de prestige », qui connaît des caractéristiques régionales. Elle est associée aux «Blancs », mais se diffuse en réalité au-delà de ce groupe ;

- la « variété endolingue non prestigieuse » attribuée aux « Créoles » et aux «non-Blancs »

- la «variété néo-francophone» de familles indo-mauriciennes, créoles ou chinoises considérées comme de nouveaux bourgeois et qui transmettent le français qui n'est pas leur L1 à leurs enfants.

${ }^{9}$ Ce phénomène n'est pas réservé aux 5 locuteurs, nous avons dans les enregistrements quelques jeunes faisant plus ou moins partie du G5 ayant les mêmes usages lors d'interactions avec un membre du G5.

${ }^{10}$ Même si ce facteur a déjà été mis en avant plus haut pour les situations de relâchement chez Farah et le père de Coralie qui influent sur l'apparition de $b e$.

${ }^{11}$ Nous traduisons sipa kwa ici par « et tout » pour nous rapprocher du contexte même si une traduction littérale serait « je ne sais quoi ».

${ }^{12}$ Idem.

${ }^{13}$ La traduction a été effectuée selon ce qui nous paraissait le plus proche en français en essayant de nous adapter au contexte.

${ }^{14}$ Nous avons choisi cet extrait car be et ben apparaissent tous les deux ainsi que sipa kwa. La distribution de be pour le discours rapporté d'Amélie et de ben pour le discours rapporté de son petit-ami Anthony n'est que fortuite, elle utilise dans le reste de l'enregistrement les deux peu importe la personne dont elle rapporte le discours.

${ }^{15}$ Ici, le groupe imité n'est pas clair, il peut s'agir des Blancs de Maurice comme des Français de France, contrairement à l'extrait cité plus haut de Dorian et ses cousins dans lequel l'imitation de Jean des Blancs de Maurice est plus explicite. Il n'en reste pas moins que cet usage, apparenté au niveau prosodique nous indique que la locutrice « emprunte une autre voix ».

${ }^{16}$ Nos enregistrements ont été transcrits à l'aide du logiciel Praat et les analyses d'occurrences ont été effectuées avec le logiciel libre d'accès AntConc. 